\title{
Mammary-Type Myofibroblastoma
}

National Cancer Institute

\section{Source}

National Cancer Institute. Mammary-Type Myofibroblastoma. NCI Thesaurus. Code C121181.

A benign mesenchymal neoplasm characterized by the presence of spindle shaped myofibroblasts and mast cells in a collagenous stroma. It is histologically identical to the myofibroblastoma of breast. It usually arises from the subcutaneous tissue and the most common sites of involvement are the inguinal/groin, paratesticular, and vulvovaginal areas. 